\title{
Unsteady Pressure Fluctuations in an Inducer*
}

\author{
Mitsuru SHIMAGAKI**, Tomoyuki HASHIMOTO ${ }^{* *}$, Mitsuo WATANABE ${ }^{* *}$, \\ Satoshi HASEGAWA**, Noriaki NAKAMURA*** and Takashi SHIMURA**
}

\begin{abstract}
In this investigation, pressure fluctuations in an inducer were observed in order to examine the characteristics of unsteady cavitation at lower cavitation coefficients. From the spectrum analysis, it was confirmed that the pressure fluctuations occurred with the frequency of $0.6 \times \omega_{s}\left(\omega_{s}\right.$, shaft rotational frequency), $1.0 \times \omega_{s}, 1.1 \sim 1.3 \times \omega_{s}$ and also $2.1 \times \omega_{s}$. The $0.6 \times \omega_{s}$ was caused by the rotating phenomena in an inducer rotational direction. The $1.1 \sim 1.3 \times \omega_{s}$ was caused by the super-synchronous rotating cavitation. The $2.1 \times \omega_{s}$ was caused by rotating cavitation in the counter direction. In addition, the amount of head reduction of the inducer caused by the synchronous cavitation $\left(1.0 \times \omega_{s}\right)$ depended on the cavity pattern of the blades.
\end{abstract}

Key Words: Inducer, Rotating Cavitation, Pressure Fluctuation, Pump, Rotating Stall

\section{Introduction}

Presently, liquid oxidizer (LOX) and liquid hydrogen (LH2) are used as propellants for the Japanese rockets engine system and a turbopump is used in order to ensure its supply pressure. In this turbopump, an inducer is mounted in its first stage to improve its pump suction performance. The inducer is required to have high suction performance. The relation between the improvement of the suction performance and the suppression ${ }^{(1)-(4)}$ of inducer instabilities caused by cavitation has been studied for the last decade.

Representative unstable phenomena of cavitation are rotating cavitation ${ }^{(5)}$ and cavitation surge ${ }^{(6)}$. Rotating cavitation is typified by:

- "Forward-traveling rotating cavitation", which rotates in an inducer rotational direction at a different speed from that of the rotating of the inducer.

- "Backward-traveling rotating cavitation ${ }^{(7) ", \text { which }}$ rotates in the counter direction of the inducer.

- "Synchronous rotating cavitation", which rotates at the same speed as the inducer.

* Received 31st March, 2006 (No. 04-0906). Japanese Original: Trans. Jpn. Soc. Mech. Eng., Vol.71, No.702, B (2005), pp.467-473 (Received 16th August, 2004)

** Turbopump Section, Institute of Aerospace Technology, Japan Aerospace Exploration Agency, 1 Koganezawa, Kimigaya, Kakuda, Miyagi 981-1525, Japan.

E-mail: simagaki@kakuda.jaxa.jp

*** Japan Technology and Science Agency, Tokyo Headquarters (Science Plaza), 5-3 Yonbancho, Chiyoda-ku, Tokyo 102-8666, Japan
While the synchronous rotating cavitation occurs, the synchronous axis vibration is enlarged, thus deteriorating the reliability of the turbopump. On the other hand, cavitation surge is a primary unstable phenomenon of the system which is caused by the dynamic response of cavity ${ }^{(6)}$. Besides the rotating cavitation and cavitation surge, there also occurs a phenomenon ${ }^{(8)}$ resembling rotating stall. These unsteady phenomena enlarge the axis vibration of the turbopump and cause stress fluctuations of the inducer blades. To cope with these problems, it is necessary to understand these characteristics.

This report examines the suction performance of a rocket engine turbopump inducer using water as working fluid, and studies unstable oscillating phenomena of cavitation.

\section{Nomenclature}

A1, A2, B1, B2 : Sensor positions

$P_{s}:$ Inducer inlet static pressure $(\mathrm{Pa})$

$P_{v}:$ Saturated vapor pressure $(\mathrm{Pa})$

$Q:$ Flow rate $(1 / \mathrm{s})$

$Q_{d}:$ Design flow rate $(1 / \mathrm{s})$

$Q / Q_{d}$ : Specific flow rate

$W_{i}:$ Inducer relative velocity $(\mathrm{m} / \mathrm{s})$

$\Delta C_{P A 1}$ : Non dimension pressure fluctuation of cross section A (peak to peak value), $=\Delta P_{A 1} /\left(\rho W_{i}^{2}\right)$

$\Delta P_{A 1}$ : Pressure fluctuation of cross section A (peak to peak value), (Pa)

$\triangle C_{P B 1}$ : Non dimension pressure fluctuation of cross section $\mathrm{B}$ (peak to peak value), $=\Delta P_{B 1} /\left(\rho W_{i}^{2}\right)$

$\Delta P_{B 1}$ : Pressure fluctuation of cross section B (peak to 


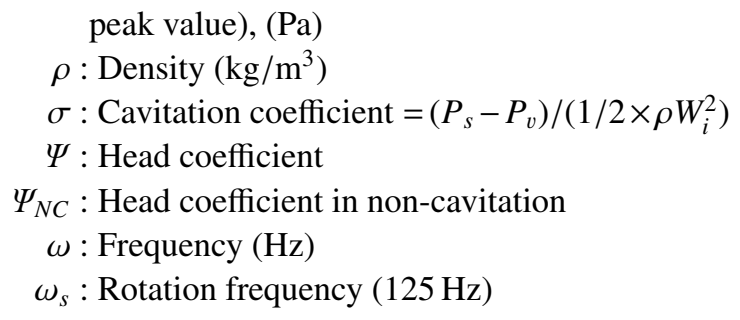

\section{Test Facility and Procedure}

\subsection{Experimental apparatus}

The present experiment was conducted using a closed-loop inducer test facility. The schematic diagram of the facility is shown in Fig. 1. After water was deaerated in a vacuum tank, the deaerated water was poured into the tunnel. The inducer is powered by the $185 \mathrm{~kW} \mathrm{DC}$ motor through a multiplying gear box. A pressurizationdepressurization piston was installed in the upstream of the inducer in order to adjust the pressure at the inducer inlet. A flow control valve was installed in the downstream of the inducer. The flow control valve keeps the set flow rate during the experiment constant, receiving feedback signals from the turbine flow meter. In the present experiment it is possible to keep the flow rate $Q / Q_{d}$ within $\pm 3 \%$ for even if there is a drastic drop in the inducer head. $Q$ and $Q_{d}$ mean experimental flow rate and design flow rate, respectively. The heat-exchange equipment was also installed in the downstream to keep the working fluid at a constant temperature during the experiment.

Figure 2 shows the test inducer and the position of the pressure sensors that were placed on the test inducer casing. Figure 3 describes the circumference location of the four pressure sensors placed on the inducer casing in order to identify the rotating direction of unsteady pressure fluctuation. The positions of the sensors on the inducer inlet section are called $\mathrm{A} 1$ and $\mathrm{A} 2$ and those on the inducer middle section are called B1 and B2. The attached letters indicate the circumference positions showed in Fig. 3. Sensor 2 is located in the position rotated by 90 degrees in the inducer rotational direction from the position of sen-

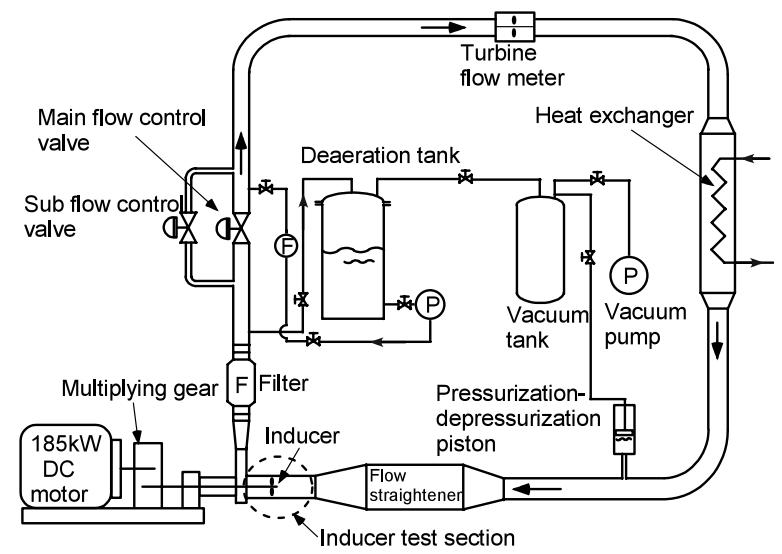

Fig. 1 Cavitation tunnel test facility sor 1 in order to identify the direction of the rotating cavitation. The test inducer has three blades.

\subsection{Procedure}

The experiment was conducted at a rotating speed of $7500 \mathrm{rpm}$. At a constant rotating speed and a constant flow rate, the cavitation coefficient $\sigma=\left(P_{s}-P_{v}\right) /(1 / 2 \times$ $\rho W_{i}^{2}$ ), was changed by reducing the pressure at the inducer inlet. The performance of the inducer was examined by the pressure at the inducer inlet and outlet, and the flow rate and the rotating speed. The measured data by the unsteady pressure sensor was recorded using A-D converter of $12 \mathrm{kHz}$ sampling frequency.

\section{Results and Discussion}

\subsection{Backward-traveling rotating cavitation}

Figure 4 shows the results of the spectrum analysis of the B1 pressure fluctuation measured in the inducer middle position at the flow rate, $Q / Q_{d}=1.03$, by changing the cavitation coefficient $\sigma$. The vertical axis shows the dimensionless pressure fluctuation $\Delta C_{P B 1}\left(=\Delta P_{B 1} /\left(\rho W_{i}^{2}\right)\right)$. The depth axis shows the cavitation coefficient $\sigma$. As shown in Fig. 4, in the region of $\sigma \fallingdotseq 0.069$ to 0.105 , the frequency of the peak was about 2.1 times rotation frequency $\omega_{s}(=125 \mathrm{~Hz})$. In the case of $Q / Q_{d}=1.06$, a pressure fluctuation of about 2.1 times of $\omega_{s}$ (hereinafter called, $2.1 \times \omega_{s}$ ) was observed in the region of $\sigma \fallingdotseq 0.070$ to 0.106 . The amplitude of this component is relatively

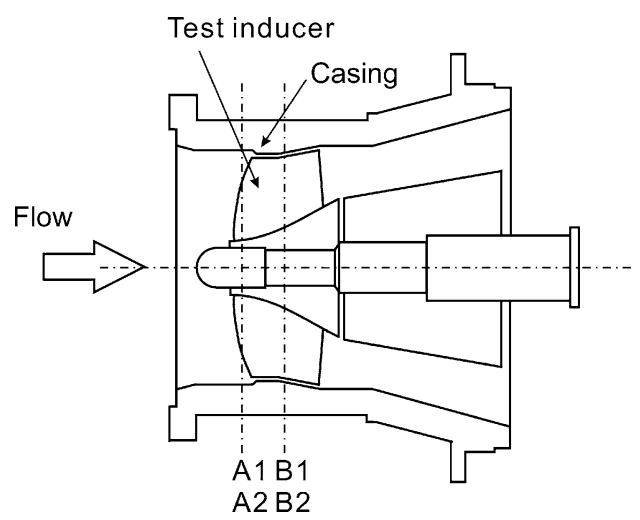

Fig. 2 Schematic diagram of test inducer and sensor locations

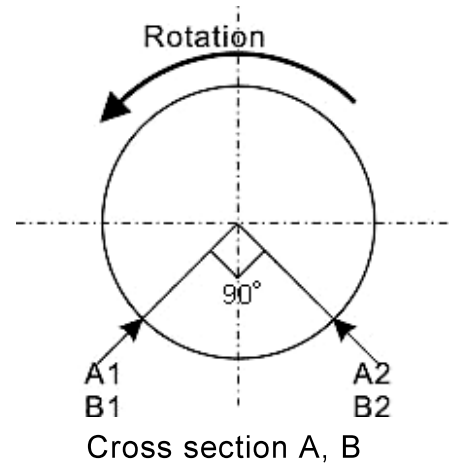

Fig. 3 Schematic diagram of sensor locations 


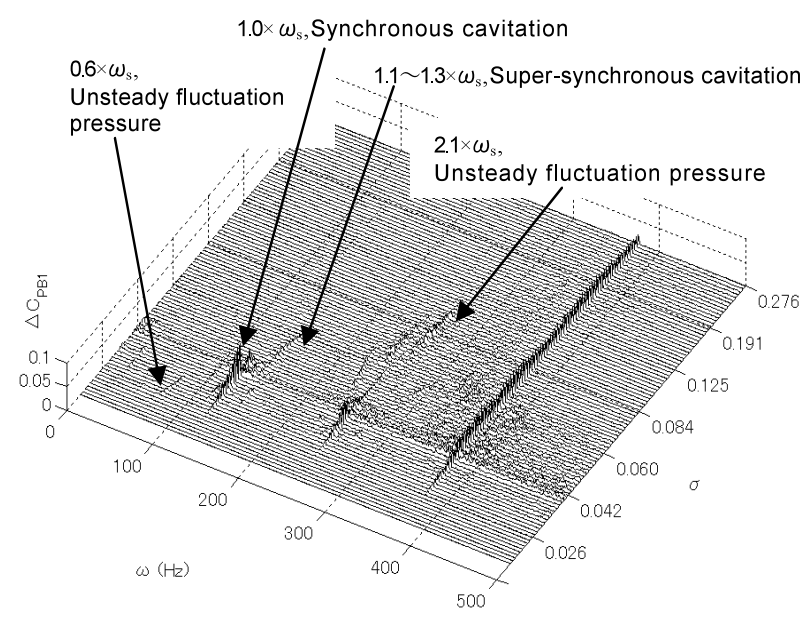

Fig. 4 Spectrum analysis of pressure fluctuation B1 for $Q / Q_{d}=1.03$

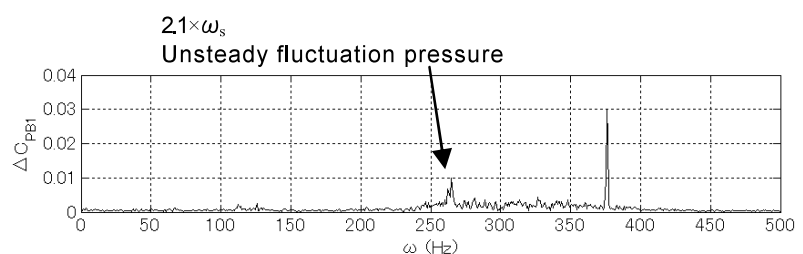

(a) B1 sensor position

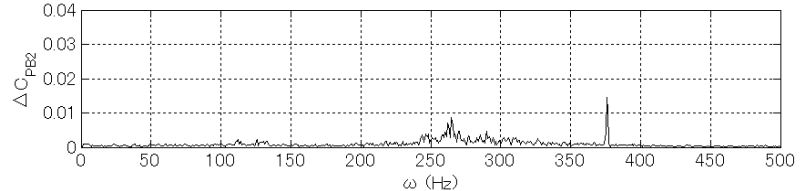

(b) B2 sensor position

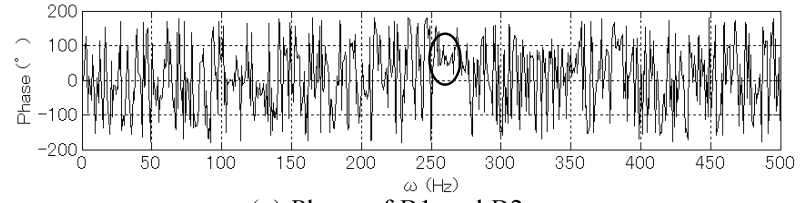

(c) Phase of B1 and B2 sensor

Fig. 5 Spectrum analysis and phase analysis of pressure fluctuation B1 and B2 for $Q / Q_{d}=1.03$

small, $20 \%$ of the maximum amplitude of synchronous rotating cavitation $\left(1.0 \times \omega_{s}\right.$, say later $)$ and this component is the same as that of the unsteady pressure fluctuation that appeared in high $\sigma$ regions. In order to examine the situation in which this unsteady pressure fluctuation occurs, the phase difference of pressure fluctuations was studied as follows.

Figure 5 (a) and (b) shows the results of spectrum analysis on sensor B1 and B2. Phase difference analysis was conducted in the region of $\sigma \fallingdotseq 0.089$ to 0.095 where $2.1 \times \omega_{s}$ component appears. Figure 5 (c) showed the results of analysis of the phase difference between B2 sensor (as shown in Fig. 5(b)) and B1 sensor (as shown in Fig. 5 (a)) on the inducer middle cross section. Since sensor 2 is located in the position rotated by 90 degrees in the inducer rotational direction from the position of sensor 1, there is a phase delay of 90 degrees in B2 from that

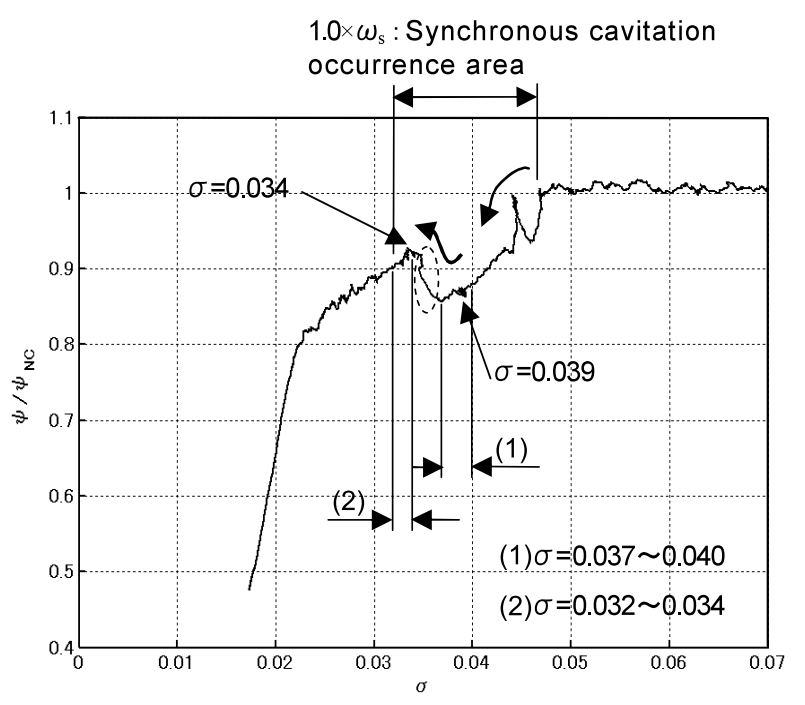

Fig. 6 Suction performance for $Q / Q_{d}=1.00$

in B1 on the inducer inlet where rotated fluctuations occur in the inducer rotational direction, while there is an opposite phase lag in the case of fluctuations moving in the counter direction. The phase difference of B2 (Fig. 5 (b)) from B1 (Fig. 5 (a)) was +60 to +70 degrees, meaning that the phase advanced by 90 degrees. As a result, it is concluded that the unsteady pressure fluctuation of $2.1 \times \omega_{s}$ is a phenomenon rotating in the reverse direction of the inducer rotation.

\subsection{Synchronous rotating cavitation}

Figure 6 shows the suction performance curve in the inducer suction test at the flow rate of $Q / Q_{d}=1.00$. The vertical axis shows the inducer head ratio $\Psi / \Psi_{N C}(\Psi$ : the inducer head coefficient, $\Psi_{N C}$ : the inducer head coefficient during non-cavitation condition) and the horizontal axis shows the cavitation coefficient $\sigma$.

As shown in Fig. 6, the inducer suction performance curve's overall trend is downward. However the curve dips at around $\sigma \fallingdotseq 0.047$ and recovers again at around $\sigma \fallingdotseq 0.035$. To inspect the cause of the dip in this curve, spectrum analysis was made on B1 pressure fluctuation in the inducer middle position.

Figure 7 shows the result of spectrum analysis of B1. The pressure fluctuation component $\left(1.0 \times \omega_{s}\right)$ caused by synchronous rotating cavitation is growing in the region from $\sigma \fallingdotseq 0.047$ to 0.032 in which a drop in $\Psi / \Psi_{N C}$ occurs as shown in Fig. 6. Regarding the $1.0 \times \omega_{s}$ component as shown in Fig. 7, there is an area where $\sigma$ is comparatively high (region of around $\sigma=0.047$ to 0.040 ), which is a transient gain in peaks of $1.0 \times \omega_{s}$ 's $\Delta C_{P B 1}$. Hereupon, the $1.0 \times \omega_{s}$ 's peaks is seems to show high peak's $\sigma$ region (1) and low peak's $\sigma$ region (2) as shown in Fig. 7. When this result is examined related to the suction performance as shown in Fig. 6, the $\sigma$ region (1) corresponds to the $\sigma$ region (around $\sigma \fallingdotseq 0.037$ to 0.040 ) where the value of $\Psi / \Psi_{N C}$ drops and the second peak's $\sigma$ region (2) corre- 


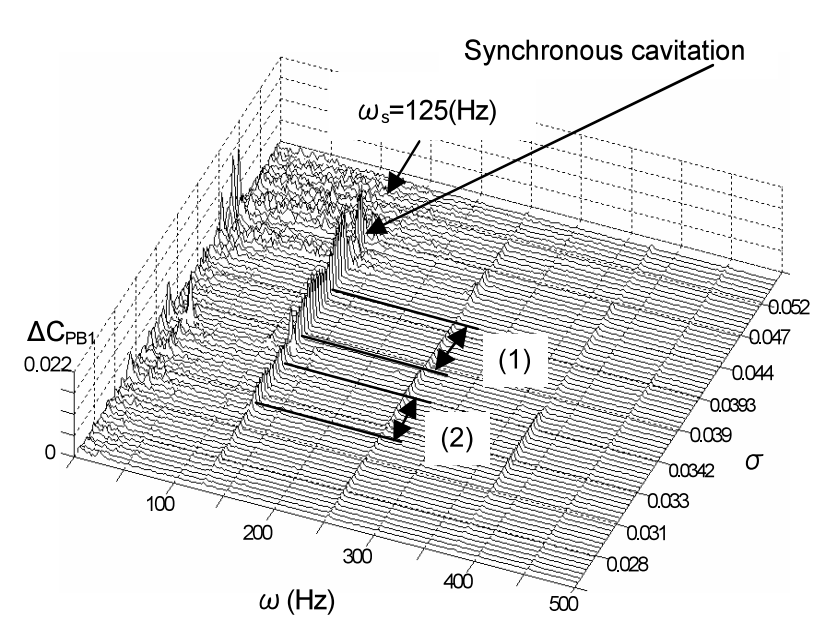

Fig. 7 Spectrum analysis of pressure fluctuation B1 for $Q / Q_{d}=$ 1.00. Cavitation number area (1) are $\sigma=0.037 \sim 0.040$ and area (2) are $\sigma=0.032 \sim 0.034$

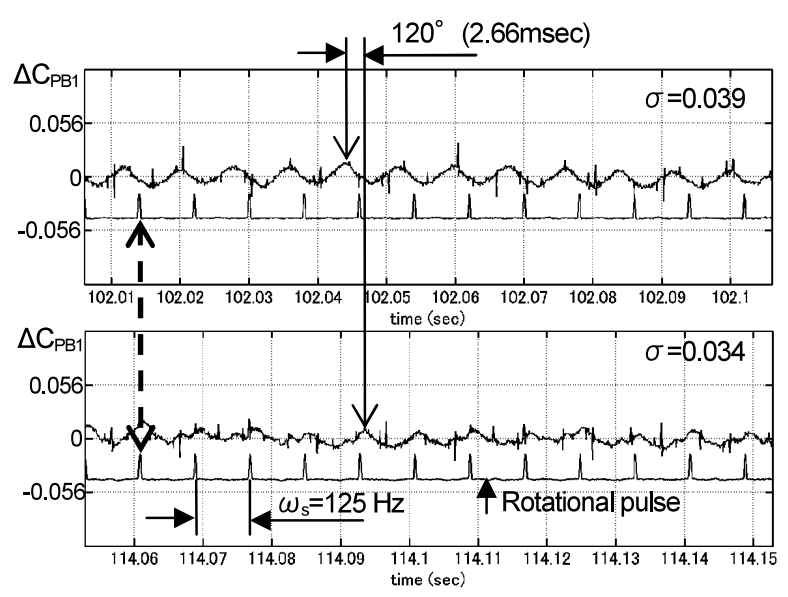

Fig. 8 Comparison of wave form of synchronous cavitation mode in case of $\sigma=0.039$ and 0.034 for $Q / Q_{d}=1.00$

sponds to the $\sigma$ region (around $\sigma \fallingdotseq 0.032$ to 0.034 ) where recovered the $\Psi / \Psi_{N C}$. The $\sigma$ region circled by a dashed line where the $\Psi / \Psi_{N C}$ is recovering corresponds to between $\sigma$ region (1) and $\sigma$ region (2) (around $\sigma \fallingdotseq 0.034$ to 0.037) as shown in Fig. 7.

Figure 8 shows the time series waveform of B1 pressure fluctuation in the middle part of the inducer at $\sigma=$ 0.039 and 0.034 in the area between (1) and (2) shown in Fig. 7, overlaid with the rotation pulse $(1 \mathrm{pulse} / \mathrm{rev} .=$ $8.00 \mathrm{msec}$ ) relatively. The horizontal axis shows the time. The frequency of the pressure fluctuation of both $\sigma$ are $125 \mathrm{~Hz}$ (period; $8.00 \mathrm{msec}$ ), meaning that they are synchronous rotating cavitations. Here it is noted that the wave of the $1.0 \times \omega_{s}$ pressure fluctuation component in $\sigma=0.039$ is shifting by $2.66 \mathrm{msec}(=1 / 3$ period) from that in $\sigma=0.034$ against the rotation pulse. This phenomenon indicates that, in the inducer with three blades (with 120 degrees of pitch angle), the unbalance of cavitation occurred on one blade at $\sigma=0.039$ changed to the next blade as one pitch difference.

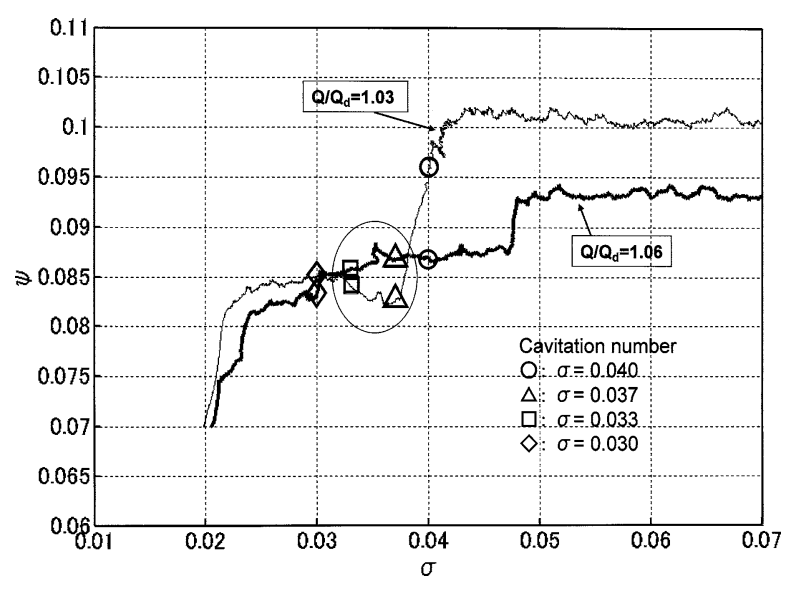

Fig. 9 Comparison of suction performances for $Q / Q_{d}=1.03$ and 1.06

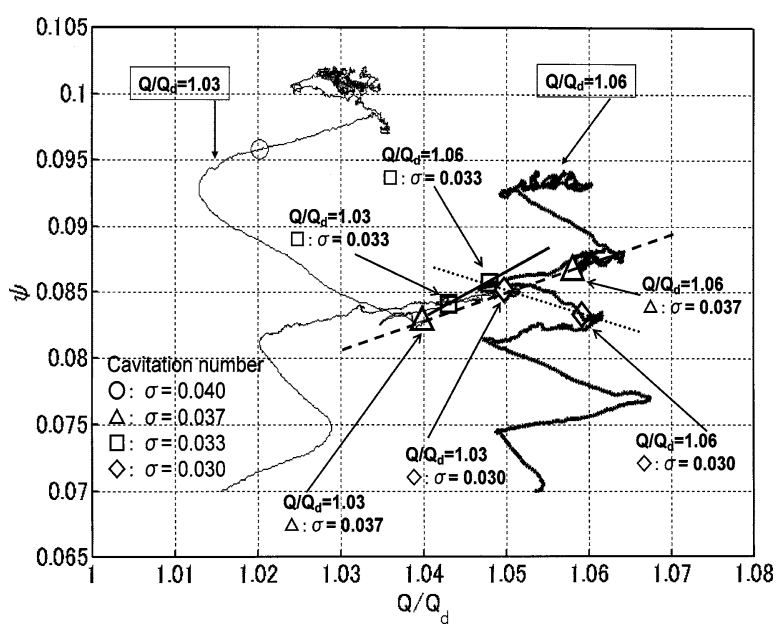

Fig. 10 Relation between head coefficient $\psi$ and flow rate ratio $Q / Q_{d}$ for $Q / Q_{d}=1.03$ and 1.06. Dashed line is in case of $\sigma=0.037$. Solid line is in case of $\sigma=0.033$. Dotted line is in case of $\sigma=0.030$

In view of this transition of cavitation unbalance and the decrease of the inducer head as shown in Fig. 6, the degree of the inducer head decreasing changes according to the blades on which cavitation unbalance occurs.

\subsection{Relation of the unsteady pressure fluctuation and the inducer head at a high flow rate}

Figure 9 shows the suction performance curve at $Q / Q_{d}=1.03$ and 1.06. The symbols in Figs. 9 and 10 describe $\diamond: \sigma=0.030, \square: \sigma=0.033, \Delta: \sigma=0.037, \bigcirc$ : $\sigma=0.040$. A sudden drop in the inducer head is observed at around $\sigma \fallingdotseq 0.041$ for $Q / Q_{d}=1.03$ and around $\sigma \fallingdotseq 0.048$ for $Q / Q_{d}=1.06$. In addition, in the region of $\sigma \fallingdotseq 0.032$ to 0.037 circled with a solid line in Fig. 9, the ups and downs of the inducer head at $Q / Q_{d}=1.03$ and 1.06 are reversed.

Figure 10 shows the movement trajectory of $Q / Q_{d}$ and $\Psi$ while the inducer head drops in under both values for around $Q / Q_{d}=1.03$ and 1.06. In the figure, the same cavitation coefficient is connected with lines. $\diamond$ : $\sigma=0.030$ connected with a dotted line, $\square: \sigma=0.033$ con- 
nected with a solid line and $\Delta: \sigma=0.037$ connected with an dashed line. In the cases of $\sigma=0.033$ and $\sigma=0.037$, the head-flow rate $\left(\Psi-Q / Q_{d}\right)$ curve makes positive slope in the region of $Q / Q_{d}=1.04$ to 1.06 . But this curve makes a negative slope in the region of $\sigma$ less than 0.030 .

Figure 4 shows the spectrum analysis of B1 pressure fluctuation in the case of about $Q / Q_{d}=1.03$ mentioned above. In the region of $\sigma \fallingdotseq 0.069$ to 0.105 , where the unsteady pressure fluctuation of $2.1 \times \omega_{s}$ occurred as mentioned in section 4.1, there were no changes observed in the inducer head, though the cavitation coefficient was higher as is shown in Fig. 9. The region where the supersynchronous rotating cavitation with lower cavitation coefficient occurs is limited to the area of $\sigma \fallingdotseq 0.062$ to $\sigma \fallingdotseq 0.046$ which is observed before the decrease in the inducer head starts as shown in Fig. 9. Accordingly, in the case of $Q / Q_{d}=1.03$, the inducer head doesn't come down in spite of the fact that unsteady pressure fluctuation of the $2.1 \times \omega_{s}$ component and super-synchronous rotating cavitation (1.1 to $\left.1.3 \times \omega_{s}\right)$ were observed. Synchronous rotating cavitation $\left(1.0 \times \omega_{s}\right)$ starts to occur at $\sigma \fallingdotseq 0.041$ and disappears at below $\sigma \fallingdotseq 0.030$. The region of $\sigma$ where synchronous rotating cavitations appears corresponds to the area where the head difference between two flow rates of $Q / Q_{d}=1.03$ and 1.06 becomes adverse temporarily as shown in Fig. 9.

In view of the process of shrinkage change of the inducer head in the case of synchronous rotating cavitation in the region of $Q / Q_{d}=1.00$, as mentioned in section 4.2, the cavitation unbalance occurred and caused the deceasing of the head in the case of $Q / Q_{d}=1.03$ but the cavitation unbalance was not large enough to let the head drop in the case of $Q / Q_{d}=1.06$. Consequently, the head in the case of $Q / Q_{d}=1.06$ was higher than that in the case of $Q / Q_{d}=1.03$ and the $\psi-Q / Q_{d}$ curve made a positive slope.

As are shown in A1's FFT analysis in Fig. 11 and as in B1's FFT analysis in Fig. 4 , in the region of $Q / Q_{d}=1.03$, unsteady pressure fluctuation of the $0.6 \times \omega_{s}$ component

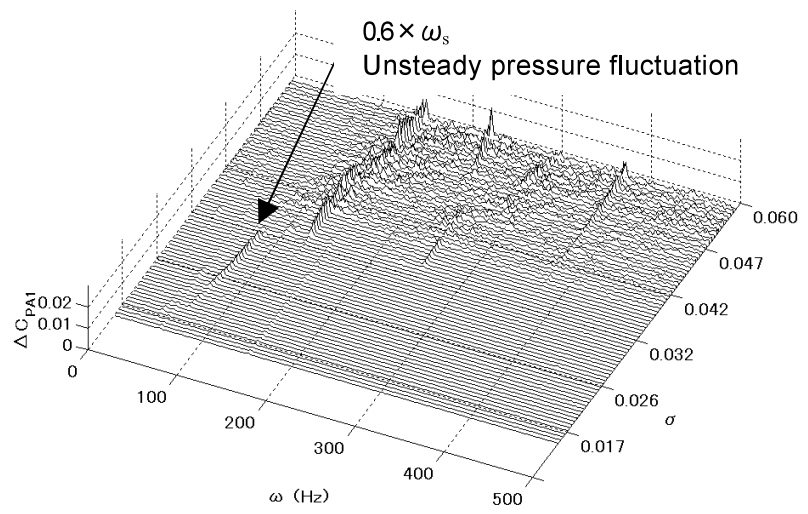

Fig. 11 Spectrum analysis of pressure fluctuation A1 for $Q / Q_{d}=1.03$ appears and the head is in a gradual decline at $\sigma \fallingdotseq 0.032$ to 0.025 (at the point of $\sigma \fallingdotseq 0.032$ the positive slope in the inducer head terminates, and at the point of $\sigma \fallingdotseq 0.025$ the head starts to drop). However, the $\Psi-Q / Q_{d}$ curve $(\diamond: \sigma=0.030)$ in the area where fluctuation of the $0.6 \times$ $\omega_{s}$ component occurs makes a negative slope as shown in Fig. 10, which is different from the characteristics of the head in the case of a rotating stall type phenomenon $\left(0.4 \times \omega_{s}\right)$ observed by Shimura ${ }^{(8)}$ et al.

Table 1 shows the phase difference observed at A1 and $\mathrm{A} 2$ sensors of the inducer inlet section and the region of the cavitation coefficient for $Q / Q_{d}=1.03$. The phase of the $0.6 \times \omega_{s}$ component ( $\sigma \fallingdotseq 0.025$ to 0.032 ) is delaying by 90 degrees. The same delay was observed in the $1.0 \times \omega_{s}$ component ( $\sigma \fallingdotseq 0.041$ to 0.044 ) which is attributed to the synchronous rotating cavitation, and in the 1.1 to $1.3 \times \omega_{s}$ component ( $\sigma \fallingdotseq 0.048$ to 0.060 ) which is attributed to the forward-traveling rotating cavitation (super-synchronous rotating cavitation). Consequently, pressure fluctuation of the $0.6 \times \omega_{s}$ component is thought to be a forward-traveling rotation in the inducer rotational direction. However, in this experiment the origin for generating the fluctuation has yet enough to be identified. A future study is expected to identify the origin.

Figure 12 shows time series data during $0.1 \mathrm{sec}-$ onds of unsteady pressure fluctuation of $0.6 \times \omega_{s}$, the synchronous rotating cavitation $\left(1.0 \times \omega_{s}\right)$ and the supersynchronous rotating cavitation ( 1.1 to $\left.1.3 \times \omega_{s}\right)$. The vertical axis shows dimensionless pressure fluctuation $\Delta C_{P A 1}$ $\left(=\Delta P_{A 1} /\left(\rho W_{i}^{2}\right)\right)$. The horizontal axis shows the time. The rotation pulse is also added in this figure. The component waveform during super-synchronous and synchronous rotating cavitation manifests as a spiky waveform, the amplitude of which is roughly 2 to 3 times larger as compared to the $0.6 \times \omega_{s}$ component. The $0.6 \times \omega_{s}$ component analyzed by the B1 pressure spectrum in Fig. 4 has about 1/10 of the maximum amplitude of synchronous rotating cavitation.

This paper has mentioned unsteady pressure fluctuations that were observed in the inducer inlet and middle

Table 1 Phase of unsteady pressure component between A1 and $\mathrm{A} 2$ for $Q / Q_{d}=1.03$

\begin{tabular}{|c|c|c|c|}
\hline $\begin{array}{c}\text { Frequency } \\
\omega\end{array}$ & $0.6 \times \omega_{\mathrm{s}}$ & $\begin{array}{c}\text { Synchronous } \\
\text { cavitation: } \\
1.0 \times \omega_{\mathrm{s}}\end{array}$ & $\begin{array}{c}\text { Super- } \\
\text { synchronous } \\
\text { cavitation: } \\
1.1 \sim 1.3 \times \omega_{\mathrm{s}}\end{array}$ \\
\hline $\begin{array}{c}\text { Cavitation } \\
\text { number } \\
\sigma\end{array}$ & $\begin{array}{c}0.025 \sim \\
0.032\end{array}$ & $\begin{array}{c}0.041 \sim \\
0.044\end{array}$ & $\begin{array}{c}0.048 \sim \\
0.060\end{array}$ \\
\hline $\begin{array}{c}\text { Phase } \\
\text { delay }\end{array}$ & $\begin{array}{c}75^{\circ} \sim \\
118^{\circ}\end{array}$ & $87^{\circ} \sim 96^{\circ}$ & $74^{\circ} \sim 84^{\circ}$ \\
\hline
\end{tabular}




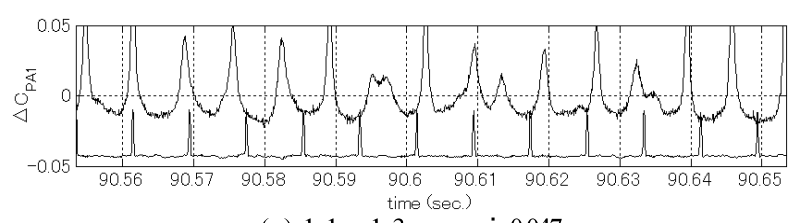

(a) $1.1 \sim 1.3 \times \omega_{s}, \sigma \doteqdot 0.047$

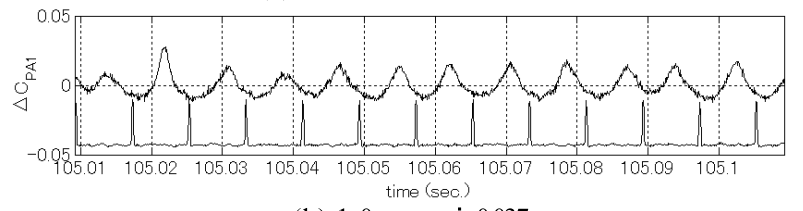

(b) $1.0 \times \omega_{s}, \sigma \doteqdot 0.037$

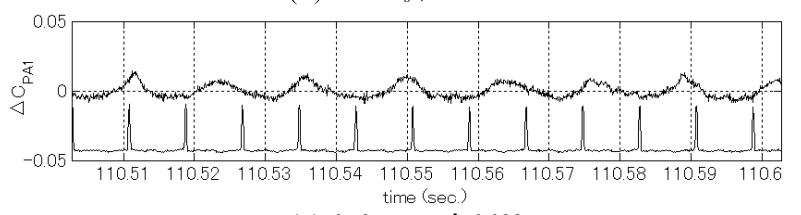

(c) $0.6 \times \omega_{s}, \sigma \doteqdot 0.032$

Fig. 12 Wave form of pressure fluctuation A1 for $Q / Q_{d}=1.03$

section, and the inducer head. Unsteady pressure fluctuation of the $2.1 \times \omega_{s}$ component, super-synchronous rotating cavitation $\left(1.1 \text { to } 1.3 \times \omega_{s}\right)^{(4),(5)}$ were not components that enormously affected the inducer head. However, synchronous rotating cavitation $\left(1.0 \times \omega_{s}\right)$ leads to drastically the inducer head breaking out at once and an increase of the synchronous shaft vibration. In contrast, in the $\sigma$ region of $0.6 \times \omega_{s}$ component, the inducer head keeps constant at a low level value.

The unsteady pressure fluctuations of $0.6 \times \omega_{s}$ and $2.1 \times \omega_{s}$ are considered to be harmful from a structural point of view, since they affect the stress of the shaft and the blades. In addition, the pressure fluctuation of synchronous rotating cavitation has more harmful from the inducer performance and the structural view points.

\section{Conclusion}

An experiment was conducted with a rocket engine turbopump-inducer using water as the working fluid. As a result of the spectrum analysis and the waveforms of pressure fluctuations that occurred in the circumference of the inducer, and in consideration of the inducer head characteristics, it is concluded that:

(1) In the past experiments ${ }^{(7)}$, a backward-traveling rotating cavitation of $1.59 \times \omega_{s}$ was observed, but in this experiment an unsteady pressure fluctuation of about $2.1 \times$ $\omega_{s}$ was observed and, as a result of phase analysis, it is concluded that this component was generated by the counter wise rotating cavitation.

(2) As for the inducer head decreasing under the synchronous rotating cavitations, it was found that the shrinkage of the head depended on the blade on which cavitation unbalance occurred.

( 3 ) It was found that in the case of $Q / Q_{d}=1.03$ synchronous rotating cavitation generated a significant head performance decrease and simultaneously the head-flow rate curve made a positive sloping.

( 4 ) Under an extremely small cavitation coefficient and with the decreasing inducer head, an unsteady pressure fluctuation of about $0.6 \times \omega_{s}$ was observed and it rotated in the inducer rotational direction. The inducer headflow rate curve showed a negative slope characteristic, unlike the rotating stall type phenomenon that showed positive slope characteristics.

\section{Acknowledgment}

The authors would like to express our gratitude to JAXA, Dr. Yoshiki Yoshida, senior researcher who gave us precious advice about this study, we also would like to extend our gratitude to Mr. Koichi Tsukahara of CosmoTec Corp. for operating machines for this experiment.

\section{References}

(1) Acosta, A.J., An Experimental Study of Cavitating Inducers, Proc. 2nd Symp. Naval Hydrodynamics, ONR/ACR-38, (1958), pp.533-557.

( 2 ) Rosenmann, W., Experimental Investigation of Hydrodynamically Induced Shaft Force with a Three Blade Inducer, Proceedings of the Symposium on Cavitation in Fluid Machinery, ASME Winter Annual Meeting, pp.172-195.

( 3 ) Kamijo, K., Shimura, T. and Tsujimoto, Y., Experimental and Analytical Study of Rotating Cavitation, ASME Paper, Vol.190 (1994), pp.33-43.

(4) Hashimoto, T., Yoshida, M., Watanabe, M., Kamijo, K. and Tsujimoto, Y., Experimental Study on Rotating of Rocket Propellant Inducer, J. Propul. Power, Vol.13 (1997), pp.488-494.

( 5 ) Kamijo, K., Shimura, T. and Watanabe, M., An Experimental Investigation of Cavitating Inducer Instability, ASME Paper 77-WA/FE-14, (1977).

( 6 ) Watanabe, M., Hasegawa, S., Hashimoto, T., Yamada, H., Komatsu, T. and Kamijo, K., Observation on Unstable Cavitation Phenomena in Rocket Turbopump Inducer, The 8th International Symposium on Transport Phenomena and Dynamics of Rotating Machinery Paper, (2000).

( 7 ) Hashimoto, T., Watanabe, M., Kamijo, K. and Tsujimoto, Y., Observations of Backward-Traveling Rotating Cavitation, (in Japanese), JSME Paper, Ser.B, Vol.63, No.605 (1997), pp.147-152.

( 8 ) Shimura, T., Yoshida, M., Kamijo, K., Uchiumi, M. and Yasutomi, Y., A Rotating Stall Type Phenomenon Caused by Cavitation in LE-7A LH2, JSME Int. J., Ser.B, Vol.45, No.1 (2002), pp.41-46. 